\title{
Infection patterns of dengue, Zika and endosymbiont Wolbachia in the mosquito Aedes albopictus in Hong Kong
}

Elaine Y.Y. Huang ${ }^{1}$, Annette Y. P. Wong ${ }^{1}$, Ivy H. T. Lee1, Zhe Qu' ${ }^{1}$ Ho Yin Yip ${ }^{1}$, Chi-wah Leung ${ }^{2}$, Shuk-may Yin² and Jerome H. L. Hui ${ }^{1 *} \mathbb{D}$

\begin{abstract}
Background: The mosquito Aedes albopictus is a vector of dengue and Zika viruses. Insecticide-resistant mosquito populations have evolved in recent decades, suggesting that new control strategies are needed. Hong Kong has a monsoon-influenced humid subtropical climate, which favours the spread of mosquitoes. However, baseline information on the composition and dynamics of the occurrence of endosymbiont Wolbachia in local Ae. albopictus is lacking, hindering the development of scientifically-informed control measures. This study identifies the presence and absence of dengue and Zika viruses, and Wolbachia infection in Aedes albopictus in Hong Kong.

Methods: Oviposition traps were set at 57 areas in Hong Kong, and both immature and adult mosquitoes were collected on a monthly basis between April 2018 and April 2019 as the study sample. Each individual mosquito in this sample was processed and screened for the presence of the dengue and Zika viruses and the endosymbionts Wolbachia wAlbA and wAlbB with PCR.

Results: Totals of 967 and 984 mosquitoes were tested respectively for the presence of dengue and Zika viruses, and no trace of either infection was found in these samples. The presence of WAlbA and WAlbB was also tested in 1582 individuals. Over $80 \%$ of these individuals were found to be stably infected with Wolbachia throughout the thirteenmonth collection period ( 47\% singly-infected; $~ 36.8 \%$ doubly infected with both wAlbA and wAlbB).

Conclusions: The high degree of Wolbachia wAlbA and wAlbB infection in Ae. albopictus mosquitoes in Hong Kong, coupled with the absence of any signs of infection by dengue and Zika viruses, contrasts significantly with the pattern of mosquito infection in other parts of Asia. Further studies of the infection pattern in local mosquitoes are warranted before mosquito control strategies used in other regions are implemented in Hong Kong.
\end{abstract}

Keywords: Aedes, Dengue, Mosquito, Wolbachia, Zika

\section{Background}

The Asian tiger mosquito (Aedes albopictus), an insect pest also known from its habits as the forest day

\footnotetext{
${ }^{*}$ Correspondence: hui.jerome@gmail.com; jeromehui@cuhk.edu.hk 1 School of Life Sciences, Simon F.S. Li Marine Science Laboratory, Key Laboratory of Agrobiotechnology, The Chinese University of Hong Kong, Hong Kong, China

Full list of author information is available at the end of the article
}

mosquito, which used to be a species confined to Southeast Asia. In recent years, however, it has spread to many other parts of the world, and can now be found in Africa, America, Europe and the Middle East. Aedes albopictus males primarily feed on nectar, while females typically feed on the blood of birds and mammals, including humans. The female Ae. albopictus often feeds by biting multiple hosts [1, 2]. Aedes albopictus is therefore an 
ideal channel for the transmission of vector-borne diseases, including dengue and Zika viruses.

Dengue virus is a single positive-stranded RNA virus of the family Flaviviridae, and can cause dengue fever (including breakbone fever, dandy fever, dengue hemorrhagic fever and dengue shock syndrome) in humans after transmission by the Aedes mosquito. The mortality rate of untreated dengue shock syndrome is more than $20 \%$, and the reported incidence of dengue fever has increased 30-fold over the past half century, resulting in 22,000 deaths annually [3]. According to current estimates, there are $\sim 390$ million dengue infections per year [4], and 3.9 billion people in 128 countries are at risk of infection [5]. In Hong Kong, the first diagnosis of dengue hemorrhagic fever was reported in 1984 [6], and it has been a statutory notifiable disease in Hong Kong since 1994 [7]. Locally acquired dengue fever has also been reported, with the first confirmed case occurring in 2003 [8]. Between 1994 and 2008 there were a total of 358 notifications, and annual numbers of notifications now range from single to double digits [7]. As modern travellers frequently move between countries, a number of cases brought in by travellers from endemic areas have also been confirmed [9-11]. The number of dengue cases reported in Hong Kong usually correlates with the occurrence of outbreaks in other Southeast Asian countries, such as those reported in 2007 or the more recent outbreak in Guangdong Province which affected 40,000 people in 2014.

The Zika virus is another single positive-stranded RNA virus of the family Flaviviridae, which can be transmitted to humans by the Aedes mosquito. Its symptoms include conjunctivitis, fever, rash, and muscle and joint pain. Most people infected by the Zika virus do not develop symptoms. Even if they do, these are generally mild. Nevertheless, Zika virus infection during pregnancy can cause microcephaly and other congenital malformations in infants, and in some cases preterm births and miscarriages. The largest documented Zika virus outbreak occurred in 2013 in French Polynesia, where 8200 cases of infection were reported in a total population of 268,000 people [12]. A total of 5168 cases associated with symptoms of the Zika virus were reported in the USA in 2016, but this figure had fallen to a mere 72 cases in the mainland USA and 148 cases in its overseas territories (Samoa, Virgin Islands, Puerto Rico, Guam and the Northern Marianas) in 2018 (Centers for Disease Control and Prevention 2019) [13]. It is not clear whether this marked decrease in the incidence of the Zika virus indicates that it is being brought under control globally, or merely in the USA and its dependencies.

Insecticides have been extensively used to control mosquito populations in recent decades, but resistance to the four main classes of neurotoxic insecticides (carbamates, organochlorines, organophosphates and pyrethroids) has gradually built up in Aedes mosquitoes in North and South America, Africa and Asia [14]. A new control strategy is presently under development, which features the use of the intracellular endosymbiotic Alphaproteobacterium Wolbachia [15]. In general, Wolbachia is prevalent in $\sim 40 \%$ of arthropod species, and the supergroups "A" ( $w \mathrm{AlbA})$ and "B" ( $w \mathrm{AlbB})$ are known to exist in mosquitoes $[16,17]$. The Wolbachia strains wAlbA and $w$ AlbB from each supergroup can be naturally found in Ae. albopictus $[18,19]$. For reasons not entirely clear, the sequences of the $16 S \mathrm{rRNA}$, wsp and $f t s Z$ genes of $w \mathrm{AlbA}$ and $w$ AlbB isolated from many parts of the world are identical, suggesting a potential lack of sequence diversity $[20-22]$. The $w$ AlbA and $w$ AlbB strains are maternally inherited, and can increase the lifespan of female mosquitoes and the period during which they produce eggs [23-25]. On the other hand, when Ae. albopictus males are doubly-infected with $w$ AlbA and $w$ AlbB, high levels of cytoplasmic incompatibility (CI) can result in crosses with uninfected or single infected females, and the removal of the two strains has no effect on lifespans or mating rates and sperm capacity [23, 26, 27]. In the Ae. albopictus cell line Aa23, sub-lethal doses of antibiotic treatment have revealed a strong negative correlation between the Wolbachia strain and dengue virus [28].

Studies of the relationship between the transmission of Wolbachia and dengue virus in Ae. Albopictus have reached different conclusions. In a head-squash assay experiment, dengue virus inhibition did not occur in Wolbachia-mediated Ae. albopictus [29]. Nevertheless, a study which measured the viral loads and Wolbachia densities in different organs of Ae. albopictus where dengue virus replication took place after ingestion concluded that Wolbachia does not affect replication of dengue virus, but is able to reduce viral infection of the salivary glands and transmission [30]. Furthermore, in a study of transient infection with the Wolbachia strain wMel (from the fruit fly Drosophila melanogaster) in Ae. albopictus, transmission of dengue virus was inhibited, but no significant effects on fecundity were observed [31]. The introduction of another strain of Wolbachia (e.g. wPip from the mosquito Culex pipiens) into Ae. albopictus was also able to induce CI [32], and this finding has also been explored as offering an alternative to the traditional strategy of releasing sterile male mosquitoes. Understanding the composition and dynamics of occurrence of Wolbachia in local Ae. albopictus is therefore of fundamental importance in providing baseline information for the development of appropriate strategies to control mosquito-borne infections. Such information is currently lacking in Hong Kong, even though Ae. albopictus 
is particularly active during the territory's subtropical summers and cases of dengue fever have been reported from time to time. Our study aimed to gather more information on the presence of dengue and Zika viruses and Wolbachia wAlbA and wAlbB in Ae. albopictus at various locations in Hong Kong during a single typical year.

\section{Methods}

\section{Study area and mosquito collection}

To collect our samples, we used oviposition traps set in 57 areas (covering 18 districts) in Hong Kong by the HKSAR Government's Food and Environmental Hygiene Department (FEHD) to monitor the breeding habits of the Aedes albopictus mosquito. More than 55 oviposition traps (ovitraps) were set in each surveyed area. An ovitrap is a $10 \mathrm{~cm}$ tall black plastic container with diameters measured from $5 \mathrm{~cm}$ (base) to $6.5 \mathrm{~cm}$ (top). A brownish wooden paddle is placed inside each ovitrap, and a black lid with 4 round-openings is used as cover. Approximately $170 \mathrm{ml}$ dechlorinated tap water is contained in each ovitrap. The ovitraps were installed in outdoor locations for two consecutive weeks each month and inspected weekly. They were collected and replaced at regular intervals with fresh ovitraps in the same locations, to enable permanent surveillance to be maintained. The retrieved ovitraps were immediately checked for the presence of larvae. If eggs were present, they were kept in an FEHD laboratory for a week at room temperature to allow them to incubate and hatch into larvae. The mosquito larvae were examined under a microscope, and their species were identified. Aedes albopictus individuals, both immature and adult, were collected from 57 different outdoor locations in Hong Kong between April 2018 and April 2019 for the purpose of DNA and RNA extraction. Individuals collected from areas with an ovitrap index (= number of Aedes-positive ovitraps/total number of ovitraps retrieved from a particular area $\times 100 \%$ ) above $10 \%$ were reared to adulthood at room temperature for 3 weeks in dechlorinated tap water using a mosquito breeder (BioQuip, California, USA). Aquarium fish feeds were used as a larval diet. Adults were harvested from the mosquito breeder every day and transferred to TRIzol reagent (Ambion, Texas, USA) at $-80{ }^{\circ} \mathrm{C}$ for further analysis of the presence of the Zika virus. Individuals collected from areas with an ovitrap index below $10 \%$, along with those which had not yet reached adulthood at the end of the 3-week-incubation period, were transferred to absolute ethanol at room temperature for further analysis of potential Wolbachia infection.

\section{RNA extraction}

RNA from 967 and 984 samples for dengue and Zika virus tests respectively was extracted using TRIzol reagent (Ambion), in accordance with the manufacturer's instructions. Both gel electrophoresis and a NanoDrop measurement were employed for quality and quantity checks, to confirm the integrity and amount of the RNA extracted.

\section{Dengue virus test}

Extracted RNA from individual samples was reverse transcribed into cDNA using the iScript ${ }^{\mathrm{TM}}$ cDNA Synthesis Kit (Bio-rad, California, USA), in accordance with the manufacturer's instructions. This involved 300-1500 ng of RNA template, a $2 \mu \mathrm{l} 5 \times$ reaction mix and $0.5 \mu \mathrm{l}$ reverse transcriptase. Polymerase chain reaction (PCR) detection was carried out in accordance with a procedure described in previous studies, using dengue specific primers (DenF: $5^{\prime}$-TCA ATA TGC TGA AAC GCG CGA GAA ACC G-3'; DenR: 5'-TTG CAC CAA CAG TCA ATG TCT TCA GGT TC-3') [33-35]. Each reaction mix includes $2 \mu \mathrm{l}$ cDNA template, $1 \times$ PCR buffer, $0.8 \mathrm{mM}$ of dNTPs, $1.5 \mathrm{mM}$ of $\mathrm{MgCl}_{2}, 0.4 \mu \mathrm{M}$ of each forward and reverse primer, $11.2 \mu \mathrm{l} \mathrm{dd} \mathrm{H} 2 \mathrm{O}$ and 1 unit of Taq DNA polymerase, with the following parameters: 1 cycle of $3 \mathrm{~min}$ at $95^{\circ} \mathrm{C}, 40$ cycles of $30 \mathrm{~s}$ at $95^{\circ} \mathrm{C}, 30 \mathrm{~s}$ at $55^{\circ} \mathrm{C}$ and $35 \mathrm{~s}$ at $72{ }^{\circ} \mathrm{C}$, and a final extension step at $72{ }^{\circ} \mathrm{C}$ for 5 mins. The amplified PCR products were examined using gel electrophoresis.

\section{Zika virus test}

Extracted RNA was also amplified into cDNA by the same process described already. The 91-bp Zika virus isolate 1_0016_PF polyprotein gene region was targeted and amplified with the specific primers Zika4481 (5'CTG TGG CAT GAA CCC AAT AG-3') and Zika4552c (5'-ATC CCA TAG AGC ACC ACT CC-3'). A total volume of $15 \mu \mathrm{l}$ was set for each reaction, including $0.3 \mu \mathrm{M}$ for each primer, $5 \mu \mathrm{l}$ cDNA template, $7.5 \mu \mathrm{l}$ master mix (iTaq Universal SYBR Green Supermix) and $1.5 \mu \mathrm{ldd}$ $\mathrm{H}_{2} \mathrm{O}$. Amplification was performed on a real-time PCR machine (CFX96; Bio-rad). In addition, a set of Zika standards with known concentrations was prepared to develop a standard amplification curve each time. A negative control was set by replacing the cDNA template with water. The quantification cycle (Cq value) was set at $0.00001 \mathrm{pg} / \mu \mathrm{l}$. The thermal cycling conditions were: (i) 1 cycle of $95^{\circ} \mathrm{C}$ for $3 \mathrm{~min}$; (ii) 39 cycles of $95^{\circ} \mathrm{C}$ for $10 \mathrm{~s}$, followed by $55^{\circ} \mathrm{C}$ for $10 \mathrm{~s}$ and $72{ }^{\circ} \mathrm{C}$ for $15 \mathrm{~s}$; and (iii) a final extension step at $72{ }^{\circ} \mathrm{C}$ for $15 \mathrm{~s}$. Three technical replicates were conducted for each sample.

\section{DNA extraction}

DNA from 1582 samples of Wolbachia was extracted using the Purelink Genomic DNA Mini Kit (Invitrogen, California, USA), with slight modifications to the 
manufacturer's instructions. Individual larval or adult mosquitoes were homogenized in digestion buffer with proteinase $\mathrm{K}$ and incubated at $55{ }^{\circ} \mathrm{C}$. After digestion, samples were centrifuged at $14,000 \times \mathrm{rpm}$ for $3 \mathrm{~min}$, and the supernatant was then transferred for further DNA extraction. To remove any RNA and isolate DNA effectively, RNase A was also added and incubated for $2 \mathrm{~min}$ at $37^{\circ} \mathrm{C}$. The quality and integrity of the extracted DNA was determined by gel electrophoresis and observed with the Gel Doc ${ }^{\mathrm{TM}}$ EZ imager (Bio-rad).

\section{Wolbachia wAlbA and wAlbB tests}

PCR was used to amplify the targeted DNA fragment of $w$ AlbA and $w$ AlbB. Amplification was carried out on a $\mathrm{T}_{100}{ }^{\mathrm{TM}}$ thermocycler (Bio-rad) with the following parameters: 1 cycle of $3 \mathrm{~min}$ at $95^{\circ} \mathrm{C} ; 40$ cycles of $30 \mathrm{~s}$ at $95^{\circ} \mathrm{C}$, $30 \mathrm{~s}$ at $56^{\circ} \mathrm{C}$ and $35 \mathrm{~s}$ at $72{ }^{\circ} \mathrm{C}$; and a final extension step at $72{ }^{\circ} \mathrm{C}$ for $5 \mathrm{~min}$. The final volume of each reaction was $20 \mu \mathrm{l}$, including $2 \mu \mathrm{l}$ DNA sample, $1 \times$ buffer, $0.8 \mathrm{mM}$ of dNTPs, $1.5 \mathrm{mM}$ of $\mathrm{MgCl}_{2}, 0.4 \mu \mathrm{M}$ of each forward and reverse primer, $11.2 \mu \mathrm{ldd} \mathrm{H}_{2} \mathrm{O}$ and 1 unit of Taq DNA polymerase. The forward primers used for amplifying the $w$ AlbA and $w$ AlbB DNA fragments were $\left(5^{\prime}\right.$-CCA GCA GAT ACT ATT GCG AAC AGT T-3') and (5'-AAG GAA CCG AAG TTC ATG ATC CT-3'), respectively; together with a common reverse primer (5'-AAA AAT TAA ACG CTA CTC CAG CTT CTG C-3') used in respective PCR reactions [19]. Reactions containing only water instead of DNA samples were set as negative controls. The amplified DNA fragments of $w$ AlbA and $w$ AlbB were $379 \mathrm{bp}$ and $501 \mathrm{bp}$, respectively, and their presence was checked under gel electrophoresis.

Whenever a sample showed negative results (i.e. indicating the probable absence of Wolbachia infection), another PCR amplification on the $12 S \mathrm{rDNA}$ gene was conducted using primers 12SAI (5'-AAA CTA GGA TTA GAT ACC CTA TTA T-3') and 12SBI (5'-AAG AGC GAC GGG CGA TGT GT-3') [36, 37] to retest the quality of DNA extraction. Samples which failed to amplify the $12 S$ rDNA gene were excluded from the data analyses. The PCR amplification process was performed much as in the previous procedure, but with slight modifications: 1 cycle of $3 \mathrm{~min}$ at $95^{\circ} \mathrm{C} ; 10$ cycles of $30 \mathrm{~s}$ at $95^{\circ} \mathrm{C}, 30 \mathrm{~s}$ at $50{ }^{\circ} \mathrm{C}$ and $35 \mathrm{~s}$ at $72{ }^{\circ} \mathrm{C} ; 25$ cycles of $30 \mathrm{~s}$ at $95^{\circ} \mathrm{C}, 30 \mathrm{~s}$ at $59^{\circ} \mathrm{C}$ and $35 \mathrm{~s}$ at $72{ }^{\circ} \mathrm{C}$; and a final extension step at $72{ }^{\circ} \mathrm{C}$ at $5 \mathrm{~min}$. As in the previous procedure, the amplification of $12 S \mathrm{rDNA}$ gene fragments was determined by gel electrophoresis.

\section{Statistical tests}

A Chi-square test was performed to determine whether there were significant differences between the frequency of infection in Hong Kong's eighteen separate districts. Results were considered for the $P$ values $<0.05,<0.01$, and $<0.001$.

\section{Results}

\section{Distribution of samples collected}

A breakdown of the numbers of tested samples collected from different locations in Hong Kong during the period of April 2018 to April 2019 is given in Fig. 1. Almost half of the samples were obtained in July 2018, at the peak of the breeding season for local mosquitoes. There was a significant drop in the number of samples collected in August 2018, probably because additional mosquito controls were deployed in that month to tackle a local outbreak of dengue fever in Hong Kong. Detailed information on the samples collected in different areas in Hong Kong is given in Additional file 1: Tables S1-S8.

\section{Dengue virus and Zika virus tests}

The 967 and 984 mosquito samples tested respectively for the presence of the dengue and Zika viruses showed no evidence of the presence of either virus. None of the samples tested contained more than $0.00001 \mathrm{pg} / \mu \mathrm{l}$, indicating that both viruses were absent.

\section{Wolbachia wAlbA and wAlbB tests}

Figure 1 and Table 1 illustrate the number of mosquito samples exhibiting respectively the presence of $w \mathrm{AlbA}(\mathrm{A})$, the presence of $w \mathrm{AlbB}(\mathrm{B})$, the presence of both $w$ AlbA and $w$ AlbB (A \& B), and the absence of both $w$ AlbA and $w$ AlbB (negative). Forty-one samples with DNA degradation which tested negative upon $12 \mathrm{~S}$ rDNA amplification were excluded from the analyses. Over $80 \%$ of the mosquito samples tested were found to be Wolbachia-infected. $36.8 \%$ of the samples were doubly infected with both $w$ AlbA and $w$ AlbB. $23 \%$ were singly infected with $w$ AlbA, and $24 \%$ with $w$ AlbB. Detailed month-to-month results are shown in Additional file 2: Figures S1-S12.

The geographical distribution of Wolbachia infection in Hong Kong mosquitoes varied across the territory's 18 districts (Fig. 2). The area with the highest Wolbachia infection rate was the urban district of Kwun Tong in Kowloon (District number 6). 94.9\% of mosquito samples collected from Kwun Tong were Wolbachia-infected, compared with only $56.3 \%$ of the samples collected from the New Territories district of Tai Po (District number 13).

Overall, the infection rate of either $w$ AlbA or $w$ AlbB ranged between $12-47.4 \%$, while the infection rate among mosquitoes doubly infected with both $w$ AlbA and $w$ AlbB was between $14.6-58.9 \%$. In the Chi-square 
analyses, it was also found that the frequency of infection differed significantly among the 18 districts $\left(\chi^{2}=74.5\right.$, $d f=17, P<0.05)$. As most mosquitoes can fly some distance away from their breeding sites, widening the potential area of infection, the data are also presented according to three broader geographical divisions: (i) the New Territories and Kowloon; (ii) the Outlying Islands; and (iii) Hong Kong Island. These three areas are separated by bodies of seawater (Fig. 3).

\section{Discussion}

Aedes albopictus has long been known to be a vector of dengue and Zika viruses in many parts of Southeast Asia. The mosquito can be infected either with single (wAlbA or wAlbB) or multiple (wAlbA and wAlbB) strains of Wolbachia. This study reveals the infectious status of dengue and Zika viruses, and Wolbachia in mosquitoes in Hong Kong, and reveals markedly different dynamics to those reported previously in other parts of Asia.

The current measures for mosquito control in Hong Kong were established by the HKSAR Government in 2004, when a surveillance programme using ovitraps to monitor the distribution of Ae. albopictus in different areas was introduced. As the female mosquito lays her eggs near (as opposed to directly in) water, and has a short flight range (less than two hundred metres), the ovitraps used in this study should provide a representative sample of mosquito distribution across Hong Kong's various districts at the time of the study.

Our results document a high infection rate $(>80 \%)$ of Wolbachia in Ae. albopictus in Hong Kong. This finding concurs with other published data showing the general trend of natural infection of Wolbachia in Ae. albopictus $[18,20,23,38]$. It contrasts markedly with the low infection rate $(25 \%, 71$ out of 284 samples) documented in a recent study conducted in Malaysia [35], but is in line with the higher rate found by other studies $(94.8 \%, 271$ out of 286 samples) carried out both in Malaysia [39] and in Thailand (100\%, 1081 out of 1081 samples) [23].

The infection rate of the dengue virus in Malaysia was also investigated in Teo's study [35], and was found to be $25.7 \%$ (73 out of 284 samples). By contrast, our own study found no evidence for the existence of dengue virus in Hong Kong (0 out of 967 samples).

While the study by Joanne et al. [39] in Malaysia found a high Wolbachia infection rate in Ae. albopictus, 91.6\% of its samples were superinfected with both wAlbA and wAlbB, and very few were singly infected $(\sim 1 \%$ with wAlbA and $\sim 2 \%$ with wAlbB). A similar pattern has also been documented in Thailand, where $99.41 \%$ of samples were doubly infected with both Wolbachia strains) [23]. Although the rate of double infection was also found to be higher than that of single infection in all eighteen districts in Hong Kong, around $40 \%$ of our own mosquito samples were singly infected. Assuming the observed differences are not built on the different methodologies employed in different studies, these data suggest that the composition of Wolbachia could be different in Ae. albopictus in different parts of Southeast Asia. This phenomenon is comparable to the pattern of Wolbachia infection in tsetse flies, where infection rates vary from region to region [40].

The dynamics of Wolbachia composition remain relatively constant during mosquito's breeding season (April to October) in Hong Kong. The striking local Wolbachia infection pattern of Ae. albopictus in Hong Kong ( $47 \%$ of samples singly-infected, and $\sim 36.8 \%$ doubly infected with wAlbA and wAlbB) remained relatively stable over the study period. Further studies in a few years' time would enable us to verify whether this composition has undergone any change.

Detailed statistical analyses for Wolbachia infection were also carried out and shown in Additional file 1: Tables S5-S8. Differences were found between the infection rates in Kowloon and New Territories to the islands. In particular, mosquitoes in District numbers 6, 13 and 17 (Kwun Tong, Tai Po and Yuen Long) have different infection rates than expected values. Considering that 64 to 92 mosquitoes were taken from each of these three districts, future follow-up work will also be required to determine whether this is an artifact of sample size, or whether this represents the human population/ecology of these districts.

In the past few decades, vector control methods have become heavily dependent on the use of insecticides, and resistance to insecticides in Ae. albopictus is increasingly giving grounds for concern [41]. Given the cytoplasmic incompatibility caused by Wolbachia bacteria on Aedes mosquitoes, elimination programs using Wolbachia-infected mosquitoes to replace natural mosquito populations have been carried out in several countries, including Australia, Brazil, Colombia, Indonesia and Vietnam [15]. In some parts of Asia, programs have also been developed which involve the release of genetically modified mosquitoes.

Against this background, it is worth considering whether a case exists for the introduction of alternative mosquito control methods in Hong Kong. It is not yet clear whether the zero infection rate of both dengue and Zika viruses in Ae. albopictus in Hong Kong reflects the consequences of Wolbachia infection, the effectiveness of the government's program for the regular application of insecticides, changes in the genetics of the mosquito population, or a combination of these causes. Alternatively, these viruses may be present in the local mosquito 
No. of tested samples between 2018 and 2019

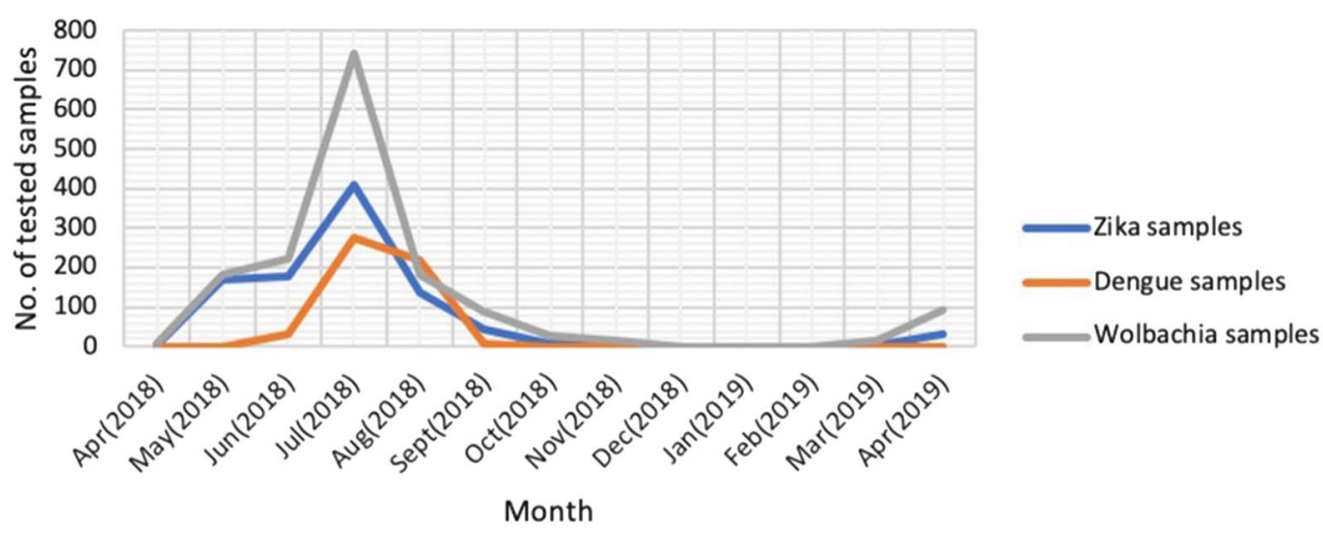

\section{Monthly number of different Wolbachia infection frequency between 2018 and 2019}

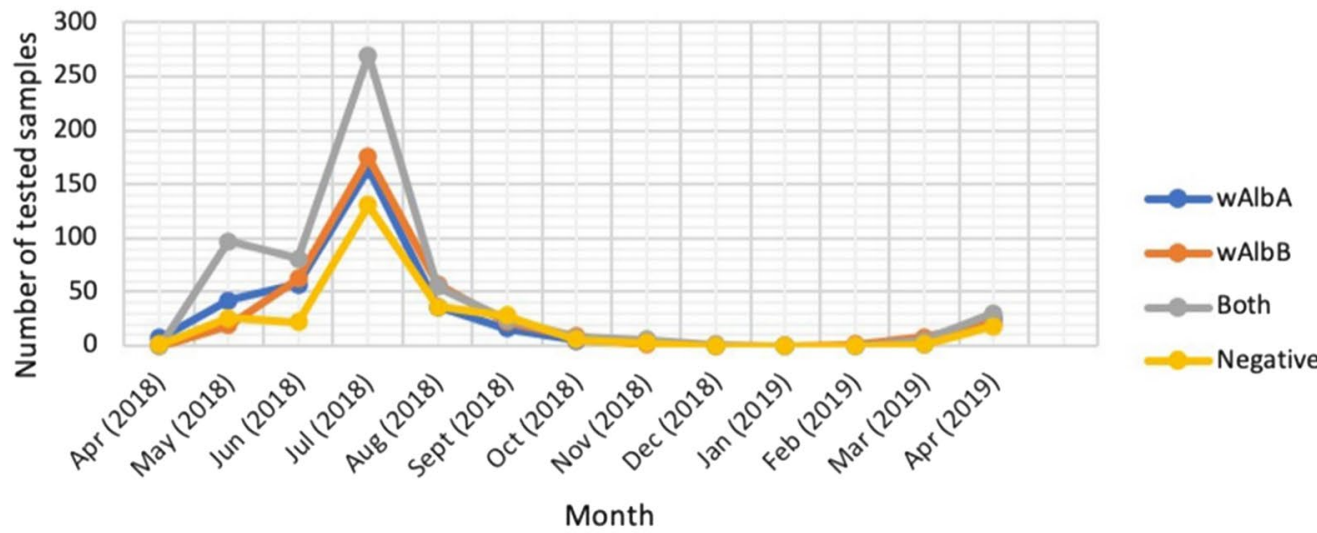

Monthly number of tested samples from Hong Kong 18 districts between 2018 and 2019



Fig. 1 Graphs showing the number of individual mosquitoes tested for the dengue and Zika viruses and for Wolbachia (upper panel); Wolbachia infection frequency (middle panel), and tested samples from different districts (lower panel) between 2018 and 2019 
Table 1 Number of individual Ae. albopictus mosquitoes found to be infected with Wolbachia wAlbA and wAlbB strains during the study period (April 2018 to April 2019)

\begin{tabular}{|c|c|c|c|c|c|c|}
\hline Year & Month & wlbA & wlbB & wlbA \& wlbB & Negative & Total \\
\hline \multirow[t]{9}{*}{2018} & April & 7 & 0 & 0 & 1 & 8 \\
\hline & May & 42 & 19 & 97 & 26 & 184 \\
\hline & June & 57 & 62 & 81 & 22 & 222 \\
\hline & July & 164 & 175 & 270 & 131 & 740 \\
\hline & August & 36 & 57 & 55 & 36 & 184 \\
\hline & September & 16 & 22 & 24 & 28 & 90 \\
\hline & October & 5 & 9 & 8 & 6 & 28 \\
\hline & November & 4 & 1 & 6 & 3 & 14 \\
\hline & December & 0 & 0 & 1 & 0 & 1 \\
\hline \multirow[t]{4}{*}{2019} & January & 0 & 0 & 0 & 0 & 0 \\
\hline & February & 0 & 1 & 0 & 0 & 1 \\
\hline & March & 2 & 8 & 5 & 1 & 16 \\
\hline & April & 25 & 21 & 30 & 18 & 94 \\
\hline Total & & 358 (22.5\%) & 375 (23.5\%) & 577 (36.8\%) & $272(17.2 \%)$ & 1582 \\
\hline
\end{tabular}

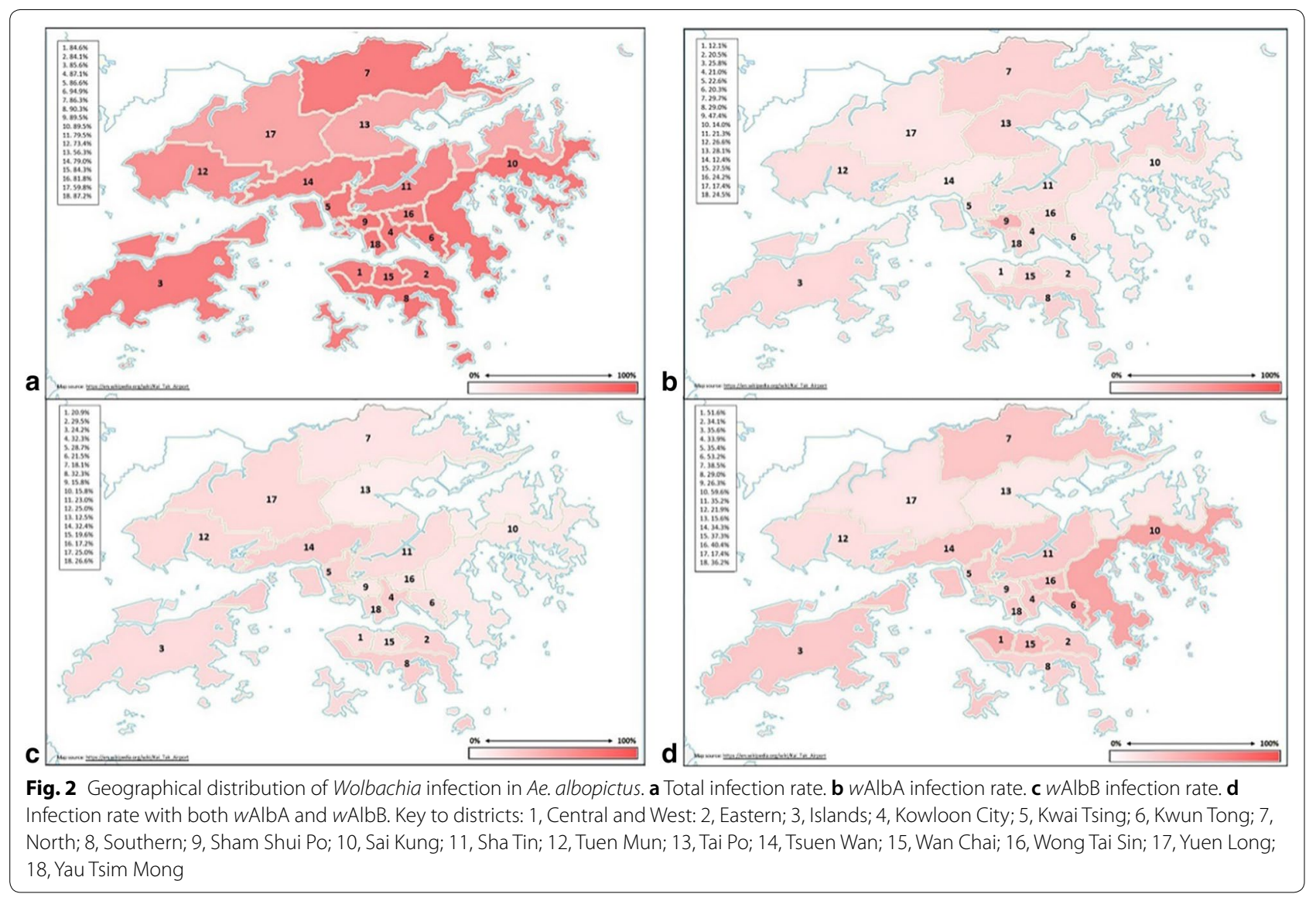




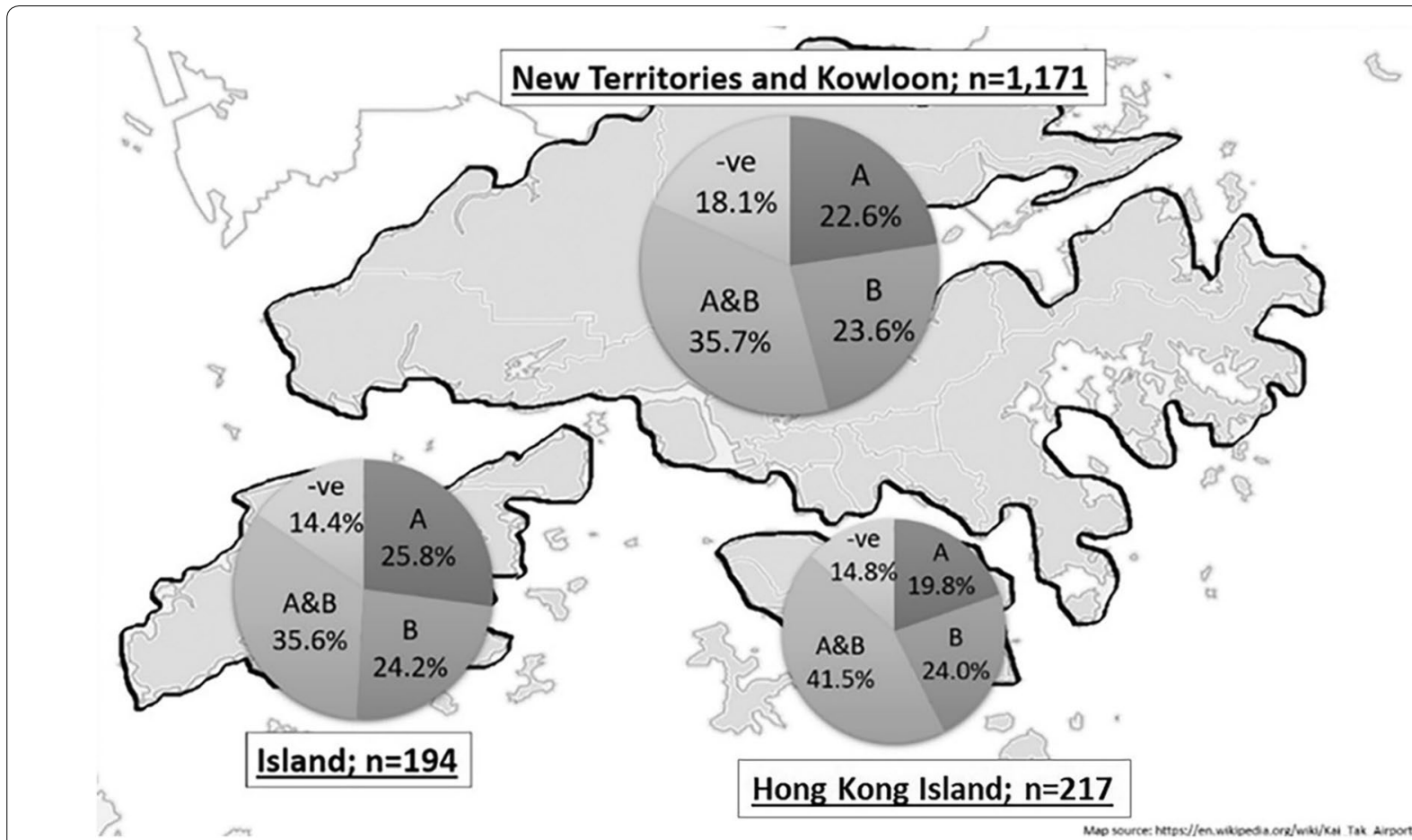

Fig. 3 Spatial distribution of Wolbachia wAlbA and wAlbB infection rates in Ae. albopictus in Hong Kong

population at such low levels that they cannot easily be detected in infected individuals. However, the latter possibility seems unlikely, as the complete absence of evidence for the existence of dengue virus in Hong Kong $(0$ out of 967 samples) contrasts markedly with the prevalence of infection found in one of the Malaysian studies (73 out of 284 samples) [35]. In any case, given that very few cases of infection with either dengue or Zika virus have occurred in Hong Kong, there is no compelling case at present for introducing alternative control methods. Nevertheless, it would be prudent to conduct further investigations to improve our knowledge of infection patterns in local mosquitoes, so that our existing control methods can be reviewed, if necessary, in the light of our growing scientific knowledge.

\section{Conclusions}

The study sheds important light on the pattern of disease-causing agents and endosymbionts in Ae. albopictus in Hong Kong over a study period that lasted just over one year. The study reveals a distinctive pattern of infection that differs in several respects to those found elsewhere in Asia.

\section{Supplementary information}

Supplementary information accompanies this paper at https://doi. org/10.1186/s13071-020-04231-x.
Additional file 1: Table S1. Sample information used in the Zika test. Table S2. Sample information used in the dengue test. Table S3. Sample information used in the Wolbachia test. Table S4. a Monthly number of tested samples between 2018 and 2019. b-f Data summary of Table S1S3. Table S5. Chi-square analyses of total infection frequency between three sites. Table S6. Chi-square analyses of different Wolbachia infection types between New Territories \& Kowloon and other sites. Table S7. Chi-square analyses of total infection frequency between 18 districts. Table S8. Chi-square analyses of different Wolbachia infection types between four districts.

Additional file 2: Figure S1. Wolbachia infection in April 2018. Figure S2. Wolbachia infection in May 2018. Figure S3. Wolbachia infection in June 2018. Figure S4. Wolbachia infection in July 2018. Figure S5. Wolbachia infection in August 2018. Figure S6. Wolbachia infection in September 2018. Figure S7. Wolbachia infection in October 2018. Figure S8. Wolbachia infection in November 2018. Figure S9. Wolbachia infection in December 2018. Figure S10. Wolbachia infection in February 2019. Figure S11. Wolbachia infection in March 2019. Figure S12. Wolbachia infection in April 2019.

\section{Abbreviations}

Cl: cytoplasmic incompatibility; ovitrap: oviposition traps; PCR: polymerase chain reaction.

\section{Acknowledgements}

The authors would like to thank Dr David Wilmshurst, former academic editor at CUHK, for editing the contents of the manuscript.

\section{Authors' contributions}

JHLH conceived and supervised the study. EYYH, AYPW, IHTL and ZQ carried out the extraction and PCR. HYY coordinated the arrangement of the samples. CWL and SMY collected the samples. EYYH and JHLH wrote the first draft of the manuscript, and all authors made detailed comments on the manuscript. All authors read and approved the final manuscript. 


\section{Funding}

This study was commissioned by the HKSAR Government's Food and Environmental Hygiene Department.

\section{Availability of data and materials \\ Not applicable.}

\section{Ethics approval and consent to participate}

Not applicable.

\section{Consent for publication}

Not applicable.

\section{Competing interests}

The authors declare that they have no competing interests.

\section{Author details}

${ }^{1}$ School of Life Sciences, Simon F.S. Li Marine Science Laboratory, Key Laboratory of Agrobiotechnology, The Chinese University of Hong Kong, Hong Kong, China. ${ }^{2}$ Pest Control Advisory Section, Food and Environmental Hygiene Department, The Government of the Hong Kong Special Administrative Region (HKSAR), Hong Kong, China.

Received: 10 February 2020 Accepted: 15 July 2020

Published online: 20 July 2020

\section{References}

1. Ponlawat A, Harrington LC. Blood feeding patterns of Aedes aegypti and Aedes albopictus in Thailand. J Med Entomol. 2005:42:844-9.

2. Farjana T, Tuno N. Multiple blood feeding and host-seeking behavior in Aedes aegypti and Aedes albopictus. J Med Entomol. 2013;50:838-46.

3. Byard RW. Lethal dengue virus infection: a forensic overview. Am J Forensic Med Pathol. 2016:37:74-8.

4. Bhatt S, Gething PW, Brady OJ, Messina JP, Farlow AW, Moyes CL, et al. The global distribution and burden of dengue. Nature. 2013;496:504-7.

5. Brady OJ, Gething PW, Bhatt S, Messina JP, Brownstein JS, Hoen AG, et al. Refining the global spatial limits of dengue virus transmission by evidence-based consensus. PloS Negl Trop Dis. 2012;6:e1760.

6. Pan HY, Chow JS. A case of hemorrhagic dengue without hypovolemia in an adult. Trop Geogr Med. 1984;36:305-7.

7. Ma T, Heywood A, Maclntyre CR. Chinese travellers visiting friends and relatives - a review of infectious risks. Travel Med Infect Dis. 2015;13:285-94.

8. Auyeung TW, Que TL, Lam KS, Ng HL, Szeto ML. The first patient with local acquired dengue fever in Hong Kong. Hong Kong Med J. 2003;9:127-9.

9. Chuang V, Wong TY, Leung YH, Ma E, Law YL, Tsang O, et al. Review of dengue fever cases in Hong Kong during 1998 to 2005. Hong Kong Med J. 2008;14:170-7.

10. Yang HM, Macoris ML, Galvani KC, Andrighetti MT, Wanderley DM. Assessing the effect of temperature on the population of Aedes aegypti, the vector of dengue. Epidemiol Infect. 2009;137:1188-202

11. Lo CL, Yip SP, Leung PH. Seroprevalence of dengue in the general population in Hong Kong. Trop Med Int Health. 2013;18:1097-102.

12. Rabaan AA, Bazzi AM, Al-Ahmed SH, Al-Ghaith MH, Al-Tawfiq JA. Overview of Zika infection, epidemiology, transmission and control measures. J Infect Public Health. 2017:10:141-9.

13. Centers for Disease Control and Prevention. Zika virus. https://www.cdc. gov/zika/index.html. Accessed 04 Nov 2019.

14. Moyes CL, Vontas J, Martins AJ, Ng LC, Koou SY, Dusfour l, et al. Contemporary status of insecticide resistance in the major Aedes vectors of arboviruses infecting humans. PLoS Negl Trop Dis. 2017;11:e0005625.

15. Bourtzis K, Dobson SL, Xi Z, Rasgon JL, Calvitti M, Moreira LA, et al. Harnessing mosquito-Wolbachia symbiosis for vector and disease control. Acta Trop. 2014;132:S150-63.

16. Werren JH, Windsor D, Guo L. Distribution of Wolbachia among neotropical arthropods. Proc R Soc Lond Ser B. 1995;262:147-204.

17. Baldo L, Werren JH. Revisiting Wolbachia supergroup typing based on WSP: spurious lineages and discordance with MLST. Curr Microbiol. 2007:55:81-7.
18. Sinkins SP, Braig HR, O'Neill SL. Wolbachia superinfections and the expression of cytoplasmic incompatibility. Proc Biol Sci. 1995;261:325-30.

19. Zhou W, Rousset F, O'Neill S. Phylogeny and PCR-based classification of Wolbachia strains using wsp gene sequences. Proc Biol Sci. 1998;265:509-15.

20. Armbruster P, Damsky WE Jr, Giordano R, Birungi J, Munstermann LE, Conn JE. Infection of New- and Old-World Aedes albopictus (Diptera: Culicidae) by the intracellular parasite Wolbachia: implications for host mitochondrial DNA evolution. J Med Entomol. 2003:40:356-60.

21. de Albuquerque AL, Magalhaes T, Ayres CF. High prevalence and lack of diversity of Wolbachia pipients in Aedes albopictus populations from Northeast Brazil. Mem Inst Oswaldo Cruz. 2011;106:773-6.

22. Kambhampati S, Rai KS, Burgun SJ. Unidirectional cytoplasmic incompatibility in the mosquito, Aedes albopictus. Evolution. 1993;47:673-7.

23. Kittayapong P, Baisley KJ, Sharpe RG, Baimai V, O'Neill SL. Maternal transmission efficiency of Wolbachia superinfections in Aedes albopictus populations in Thailand. Am J Trop Med Hyg. 2002;66:103-7.

24. Dobson SL, Marsland EJ, Rattanadechakul W. Mutualistic Wolbachia infection in Aedes albopictus: accelerating cytoplasmic drive. Genetics. 2002;160:1087-94.

25. Dobson SL, Rattanadechakul W, Marsland EJ. Fitness advantage and cytoplasmic incompatibility in Wolbachia single- and superinfected Aedes albopictus. Heredity. 2004;93:135-42.

26. Calvitti M, Moretti R, Porretta D, Bellini R, Urbanelli S. Effects on male fitness of removing Wolbachia infections from the mosquito Aedes albopictus. Med Vet Entomol. 2009;23:132-40.

27. Zouache K, Raharimalala FN, Raquin V, Tran-Van V, Raveloson LH, Ravelonandro P, Mavingui P. Bacterial diversity of field-caught mosquitoes, Aedes albopictus and Aedes aegypti, from different geographic regions of Madagascar. FEMS Microbiol Ecol. 2011;75:377-89.

28. Lu P, Bian G, Pan X, Xi Z. Wolbachia induces density dependent inhibition to dengue virus in mosquito cells. PLoS Negl Trop Dis. 2012;6:e1754.

29. Bian G, Xu Y, Lu P, Xie Y, Xi Z. The endosymbiotic bacterium Wolbachia induces resistance to dengue virus in Aedes aegypti. PLoS Pathog. 2010:6:e1000833.

30. Mousson L, Zouache K, Arias-Goeta C, Raquin V, Mavingui P, Failloux A The native Wolbachia symbionts limit transmission of dengue virus in Aedes albopictus. PLoS Negl Trop Dis. 2012;6:e1989.

31. Blagrove MS, Arias-Goeta C, Failloux AB, Sinkins SP. Wolbachia strain wMel induces cytoplasmic incompatibility and blocks dengue transmission in Aedes albopictus. Proc Natl Acad Sci USA. 2012;109:255-60.

32. Calvitti M, Moretti R, Skidmore AR, Dobson SL. Wolbachia strain w Pip yields a pattern of cytoplasmic incompatibility enhancing a Wolbachiabased suppression strategy against the disease vector Aedes albopictus. Parasites Vectors. 2012;5:254.

33. Rohani A, Aidil Azahary RA, Malinda M, Zurainee MN, Rozilawata H, Wan Najdah WMA, et al. Eco-virological survey of Aedes mosquito larvae in selected dengue outbreak areas in Malaysia. J Vector Borne Dis. 2014:51:327-32.

34. Lanciotti RS, Calisher CH, Gubler DJ, Chang GJ, Vorndam AV. Rapid detection and typing of dengue viruses from clinical samples by using reverse transcriptase-polymerase chain reaction. J Clin Microbiol. 1992;30:545-51.

35. Teo CHJ, Lim PKC, Voon K, Mak JW. Detection of dengue viruses and Wolbachia in Aedes aegypti and Aedes albopictus larvae from four urban localities in Kuala Lumpur, Malaysia. Trop Biomed. 2017:341:583-97.

36. Simon C, Franke A, Martin A. Ecology and evolutionary biology. In: Hewitt GM, Johnston AWB, Young JPW, editors. Molecular techniques in taxonomy. Berlin: Springer; 1991. p. 329-55.

37. O'Neill SL, Giordano R, Colbert AM, Karr TL, Robertson HM. 16 S rRNA phylogenetic analysis of the bacterial endosymbionts associated with cytoplasmic incompatibility in insects. Proc Natl Acad Sci USA. 1992;89:2699-702.

38. Kittayapong P, Baisley KJ, Baimai V, O'Neill SL. Distribution and Diversity of Wolbachia infections in Southeast Asian mosquitoes (Diptera: Culicidae). J Med Entomol. 2000;37:340-5.

39. Joanne S, Vythilingam I, Yugavathy N, Leong CS, Wong ML, AbuBakar S. Distribution and dynamics of Wolbachia infection in Malaysia Aedes albopictus. Acta Trop. 2015;148:38-45.

40. Doudoumis V, Tsiamis G, Wamwiri F, Brelsfoard C, Alam U, Aksoy E, et al. Detection and characterization of Wolbachia infections in laboratory and 
natural populations of different species of tsetse flies (genus Glossina) BMC Microbiol. 2012;12(Suppl. 1):S3.

41. Marcombe S, Farajollahi A, Healy SP, Clark GG, Fonseca DM. Insecticide resistance status of United States populations of Aedes albopictus and mechanisms involved. PLoS ONE. 2014;9:e101992.

\section{Publisher's Note}

Springer Nature remains neutral with regard to jurisdictional claims in published maps and institutional affiliations.
Ready to submit your research? Choose BMC and benefit from:

- fast, convenient online submission

- thorough peer review by experienced researchers in your field

- rapid publication on acceptance

- support for research data, including large and complex data types

- gold Open Access which fosters wider collaboration and increased citations

- maximum visibility for your research: over 100M website views per year

At BMC, research is always in progress.

Learn more biomedcentral.com/submissions 